Теорія Ймовір. та Матем. Статист. Вип. 74, 2006
Theor. Probability and Math. Statist.

No. 74, 2007, Pages 11-23

S 0094-9000(07)00693-X

Article electronically published on June 25, 2007

\title{
ON THE RUIN PROBABILITY OF AN INSURANCE COMPANY DEALING IN A $B S$-MARKET
}

UDC 519.21

\author{
A. V. BAEV AND B. V. BONDAREV
}

\begin{abstract}
We study a mathematical model of an insurance company that shares its capital by investing it in both stocks and bonds. The basic tool to describe the evolution of the stock price is the Ornstein-Uhlenbeck process. We construct an estimate for the ruin probability of an insurance company as a function of the initial capital. The distribution of the capital between stocks and bonds is found for which this estimate is minimal.
\end{abstract}

\section{INTRODUCTION}

We study a mathematical model of an insurance company that invests a part of its capital in stocks and the rest in bonds. The basic tool to describe the evolution of the stock price is the Ornstein-Uhlenbeck process (it is shown in [1 that this is an arbitragefree model). We assume that a constant fraction of the capital is invested in stocks; this constant is chosen to minimize the probability of ruin of the insurance company over a certain time interval. It is well known that if an insurance company does not deal in a $B S$-market, then the Lundberg estimate holds for the probability of ruin of the company over an infinite time interval; namely,

$$
\mathrm{P}\left\{\inf _{0 \leq t<+\infty} \xi_{t} \leq 0\right\} \leq e^{-R \xi_{0}}
$$

where $R>0$ is a nonzero solution of the equation $\lambda \int e^{x y} d F(y)=c x+\lambda$. Some questions related to the probability of ruin of an insurance company dealing in a $B S$-market are studied by A. Melnikov for the cases where the evolution of a risky asset is described by classical models. Melnikov [10] studies the probability of ruin of an insurance company for two cases, namely if either (1) the company invests the capital to bonds only, or (2) its investment is a mixture. The evolution of the stock price is described by the Wiener process in 10]; that is, the Samuelson model is treated in [10].

The following results are obtained in the present paper. We construct an estimate for the ruin probability of an insurance company as a function of its initial capital and find a constant distribution of the capital between investments in stocks and in bonds for which the estimate is minimal.

\section{Some REMARKS CONCERNING THE MODEL}

An investor purchasing both risky and nonrisky assets is said to deal in a $B S$-market. An insurance company is treated as an investor in this paper. In what follows we assume

2000 Mathematics Subject Classification. Primary 60E15, 60H05, 60H30; Secondary $62 \mathrm{P} 05$.

Key words and phrases. Poisson measure, stochastic integral, investor's portfolio, ruin probability. 
that the law of the evolution of a bank account process $B_{t}$ is given by

$$
d B_{t}=r B_{t} d t
$$

where $B_{0}$ is the initial capital invested in bonds. We consider the model

$$
S_{t}=S_{0} \exp \left\{\mu t-\frac{\sigma^{2}}{2} t+\sigma \eta_{t}\right\}
$$

to describe the evolution of the stock price (see [1]) where $\eta_{t}, t \geq 0$, is the OrnsteinUhlenbeck process; that is,

$$
d \eta_{t}=-\gamma \eta_{t} d t+\sigma d W_{t}, \quad \eta_{0}=0 .
$$

The Samuelson model

$$
S_{t}=S_{0} \exp \left\{\mu t-\frac{\sigma^{2}}{2} t+W_{t}\right\}
$$

is widely used [2, 3] for this purpose. Nevertheless we propose a different model that eliminates some of disadvantages of the Samuelson model. Below we mention a few of them. First, the price $S_{t}$ of a risky asset in the Samuelson model is represented as a solution of the stochastic differential equation

$$
d S_{t}=S_{t}\left[\mu d t+\sigma d W_{t}\right]
$$

and thus the local return at the moment $t, t \in[0 ; T]$, is such that

$$
\lim _{\Delta t \rightarrow 0} \frac{S_{t+\Delta t}-S_{t}}{S_{t}}=\mu d t+\sigma d W_{t} .
$$

We see that the local return contains two terms being of different nature, namely $\mu \Delta t$ is nonrandom, while $\sigma\left[W_{t+\Delta t}-W_{t}\right]$ are independent random variables. The assumption that the changes in the price are independent is not realistic from the point of view of practice. Our idea is to substitute the Ornstein-Uhlenbeck process instead of the Wiener process $W_{t}$ in the model (4). Second, it follows from (5) that the total return of a stock over an interval $[0 ; T]$ is

$$
\int_{0}^{T} \frac{d S_{t}}{S_{t}}=\mu T+\sigma W_{T}
$$

whence

$$
\mathrm{E} \int_{0}^{T} \frac{d S_{t}}{S_{t}}=\mu T, \quad \operatorname{Var} \int_{0}^{T} \frac{d S_{t}}{S_{t}}=\sigma^{2} T
$$

This means that the variance of the return coefficient of a stock over an interval $[0 ; t]$ increases with $t$. On the other hand, this phenomena is not detected by statistical analysis of several types of stocks. Moreover one can observe an irregular behavior of the square deviation of the return.

Using (2) and (3) we get

$$
S_{t}=S_{0} \exp \left\{\int_{0}^{t}\left(\mu-\gamma \eta_{s}-\frac{\sigma^{2}}{2}\right) d s+\sigma \int_{0}^{t} d W_{s}\right\} .
$$

Let

$$
d \chi_{t}=\left(\mu-\gamma \eta_{t}-\frac{\sigma^{2}}{2}\right) d t+\sigma d W_{t}
$$

Then

or

$$
d S_{0} e^{\chi_{t}}=S_{0} e^{\chi_{t}}\left(\mu-\gamma \eta_{t}-\frac{\sigma^{2}}{2}\right) d t+\frac{\sigma^{2}}{2} S_{0} e^{\chi_{t}} d t+\sigma S_{0} e^{\xi_{t}} d W_{t}
$$

$$
d S_{t}=S_{t}\left[\left(\mu-\gamma \eta_{t}\right) d t+\sigma d W_{t}\right]
$$


whence the return coefficient is given by

$$
\int_{0}^{T} \frac{d S_{T}}{S_{T}}=\int_{0}^{T} \mu d t+\int_{0}^{T}\left[-\gamma \eta_{t} d t+\sigma d W_{t}\right]=\mu T+\eta_{T}
$$

while its mean value is

$$
\mathrm{E} \int_{0}^{T} \frac{d S_{t}}{S_{t}}=\mu T
$$

Thus the variance of the return is equal to

$$
\operatorname{Var} \int_{0}^{T} \frac{d S_{t}}{S_{t}}=\operatorname{Var} \eta_{T}=\frac{\sigma^{2}}{2 \gamma}\left[1-e^{-2 \gamma T}\right]
$$

that is, the variance of the return approaches $\sigma^{2} / 2 \gamma$ and does not increase with $t$. It is proved in [1] that (2) is an arbitrage-free model and thus it can be used to describe the evolution of the price of a risky asset.

\section{Balance equations. The setting of the problem}

The following problem is considered in this paper. An insurance company, as an investor, divides the capital in a certain proportion. A fraction $0 \leq u \leq 1$ of the capital is used to purchase stocks, while the rest $0 \leq 1-u \leq 1$ is used to purchase bonds. The corresponding amounts of the capital are $u \xi_{t}$ and $(1-u) \xi_{t}$, respectively. If the price of one share of the stock at the moment $t$ is $S_{t}$, then the insurance company purchases $u \xi_{t} / S_{t}$ shares for $u \xi_{t}$ dollars, while the rest of the capital $(1-u) \xi_{t}$ is used to purchase bonds. At the moment $t+\Delta t$, a share of the stock will cost

$$
S_{t+\Delta t} \approx S_{t}\left(1+\mu \Delta t-\gamma \eta_{t} \Delta t+\sigma \Delta W_{t}\right)
$$

according to model (2), while any bond will cost

$$
(1-u) \xi_{t}(1+r \Delta t) \text {. }
$$

The wealth of the company at the moment $t+\Delta t$ consists of two parts, namely of

$$
\frac{u \xi_{t}}{S_{t}} S_{t+\Delta t}=\frac{u \xi_{t}}{S_{t}} S_{t}\left(1+\mu \Delta t+\sigma \Delta W_{t}\right)=u \xi_{t}\left(1+\mu \Delta t-\gamma \eta_{t} \Delta t+\sigma \Delta W_{t}\right)
$$

due to the investment in stocks and of

$$
(1-u) \xi_{t}(1+r \Delta t)
$$

due to the investment in bonds. We assume in what follows that the premium rate of the insurance company is constant and equals $c>0$. The accumulated claim process in the interval $(t, t+\Delta t)$ is modelled by a compound Poisson process $\varsigma_{t}$ and is given by

$$
\Delta \zeta_{t}=\sum_{k=Z_{t}+1}^{Z_{t+\Delta t}} \varsigma_{k}
$$

where $Z_{t}$ is a standard Poisson process with parameter $\lambda>0$, and the claim sizes $\varsigma_{k}$ are nonnegative identically distributed random variables that do not depend on $Z_{t}$. It is well known (see, for example, [4, [5]) that the random variable $\sum_{k=1}^{Z_{t}} \varsigma_{k}\left(\sum_{k=1}^{0} \varsigma_{k}=0\right)$ can be represented as the stochastic integral with respect to the Poisson measure. More precisely,

$$
\sum_{k=1}^{Z_{t}} \varsigma_{k}=\int_{0}^{t} \int y \nu(d y, d s)
$$


where $\nu(A, t)$ is a Poisson measure such that

$$
\mathrm{P}\{\nu(A, t)=i\}=\frac{\left[\lambda t \int_{A} d F(x)\right]^{i}}{i !} \exp \left\{-\left[\lambda t \int_{A} d F(x)\right]\right\} .
$$

Here $\mathrm{P}\left\{\varsigma_{k}<x\right\}=F(x)$ and $\mathrm{E} \nu(A, t)=\lambda t \int_{A} d F(x)$. In what follows we assume that the measure $\nu(A, t)$ and Wiener process $W_{t}$ are independent and either $c \geq a \lambda$ or $c>a \lambda$.

We have

$$
\xi_{t+\Delta t} \cong u \xi_{t}\left(1+\mu \Delta t-\gamma \eta_{t} \Delta t+\sigma \Delta W_{t}\right)+(1-u) \xi_{t}(1+r \Delta t)+c \Delta t-\int y \nu(d y, \Delta t)
$$

in view of equalities (12)-(15) where $\nu(A, \Delta t)=\nu(A, t+\Delta t)-\nu(A, t)$.

Using (17) we obtain the balance equation for the model (2):

$$
d \xi_{t}=\xi_{t}\left(u \mu+(1-u) r-u \gamma \eta_{t}\right) d t+u \xi_{t} \sigma d W_{t}+c d t-\int y \nu(d y, d t)
$$

We seek a number $0 \leq u \leq 1$ such that the estimate of the ruin probability for an insurance company over the interval $[0,+\infty)$ is minimal. The estimate should approach zero as $\xi_{0} \rightarrow+\infty$ (the zero load $c-a \lambda \geq 0$ is also possible).

\section{Solution of the Balance Equation}

Consider the process (see [6]

$$
\begin{aligned}
\xi_{t}^{0} & =\exp \left\{-[u \mu+(1-u) r] t+\frac{u^{2} \sigma^{2}}{2} t-u \eta_{t}\right\} \\
& =\exp \left\{-[u \mu+(1-u) r] t+\gamma u \int_{0}^{t} \eta_{s} d s+\frac{u^{2} \sigma^{2}}{2} t-u \sigma W_{t}\right\}
\end{aligned}
$$

whose stochastic differential is given by

$$
d \xi_{t}^{0}=\xi_{t}^{0}\left(-\left[u \mu+(1-u) r-u \gamma \eta_{t}\right] d t+u^{2} \sigma^{2} d t-u \sigma d W_{t}\right) .
$$

If

$$
\theta_{t}=\xi_{t}^{0} \xi_{t}
$$

then

$$
\begin{aligned}
d \theta_{t}= & \xi_{t}^{0}\left(\xi_{t}\left(u \mu+(1-u) r-u \gamma \eta_{t}\right)\right) d t+u \xi_{t} \sigma d W_{t}+c d t-\int y \nu(d y, d t) \\
& +\xi_{t} \xi_{t}^{0}\left(-\left[u \mu+(1-u) r-u \gamma \eta_{t}\right] d t+u^{2} \sigma^{2} d t-u \sigma d W_{t}\right)-u^{2} \sigma^{2} \xi_{t}^{0} \xi_{t} d t \\
= & \xi_{t}^{0}\left(c d t-\int y \nu(d y, d t)\right)
\end{aligned}
$$

whence

$$
\xi_{t}=\left[\xi_{t}^{0}\right]^{-1}\left[\xi_{0}+\int_{0}^{t} \xi_{s}^{0}(c-a \lambda) d s-\int y \tilde{\nu}(d y, d s)\right]
$$

where $\tilde{\nu}(A, t)=\nu(A, t)-\mathrm{E} \nu(A, t), a=\int y d F(y)=\mathrm{E}_{\varsigma_{k}}$, and $c \geq a \lambda$. 


\section{Moment InEqualities FOR THE STOCHASTIC INTEGRAL OVER A POISSON MEASURE}

The following result is well known [7]: if $\nu$ is the jump measure of a process, $\pi$ is its compensator, $\mu=\nu-\pi$, and $|\varphi| \leq C<+\infty$, then

$$
\mathrm{E}\left|\bar{\xi}_{t}\right|^{p} \leq c_{p} \mathrm{E}\left(\langle\bar{\xi}\rangle_{t}^{p / 2}+C^{p-2}\langle\bar{\xi}\rangle_{t}\right)
$$

for the process

$$
\bar{\xi}_{t}=\int_{0}^{t} \int \varphi(u, s) \mu(d u, d s)
$$

and all $p \geq 3$ where

$$
\langle\bar{\xi}\rangle_{t}=\int_{0}^{t} \int \varphi^{2}(u, s) \pi(d u, d s) .
$$

The condition $|\varphi| \leq C<+\infty$ does not hold in a number of cases; however, the moments of order $m$ of characteristic (24) are bounded for some integer $m>0$. Let

$$
\bar{\xi}_{t}=\int_{0}^{t} \int \alpha \xi_{s}^{0} \tilde{\nu}(d \alpha, d s)
$$

where

$$
\tilde{\nu}(A, t)=\nu(A, t)-\lambda t \int_{A} d F(x) .
$$

Here $\nu(A, t)$ is a Poisson measure with mean $\lambda t \int_{A} d F(x)$ and $\xi_{t}^{0}$ is defined by relation (19).

Theorem 1. Let $\mu>r>0$. If

for some integer $m>0$, then 1

$$
\mathrm{E}\left[\varsigma_{k}\right]^{2 m}<+\infty
$$

$$
\sup _{0 \leq t<+\infty} \mathrm{E}\left|\bar{\xi}_{\tau}\right|^{2 m} \leq b^{2 m}\left(\frac{\lambda C_{2 m}^{2}}{2 d}\right)^{m}\left(\left[1+\sqrt{\frac{2 d}{\lambda C_{2 m}^{2}}}\right]^{2}\right)^{m(m-1)}
$$

where $b=\left[\mathrm{E} \varsigma_{i}^{2 m}\right]^{1 /(2 m)}$ and

$$
d= \begin{cases}r+\frac{(\mu-r)^{2}}{2 \sigma^{2}(1+2 m)}, & \frac{[\mu-r]}{\sigma^{2}(1+2 m)}<1 \\ r+\frac{\sigma^{2}(1+2 m)}{2}, & \frac{[\mu-r]}{\sigma^{2}(1+2 m)} \geq 1\end{cases}
$$

Proof. Applying the generalized Itô formula [6] we get

$$
\begin{aligned}
\mathrm{E}\left|\bar{\xi}_{t}\right|^{2 m} & =\int_{0}^{t} \int \mathrm{E}\left[\left(\bar{\xi}_{s}+\alpha \xi_{s}^{0}\right)^{2 m}-\left(\bar{\xi}_{s}\right)^{2 m}-2 m\left(\bar{\xi}_{s}\right)^{2 m-1} \alpha \bar{\xi}_{s}^{0}\right] \lambda F(d \alpha) d s \\
& =\int_{0}^{t} \int \sum_{k=2}^{2 m} C_{2 m}^{k} \mathrm{E}\left(\bar{\xi}_{s}\right)^{2 m-k}\left(\alpha \xi_{s}^{0}\right)^{k} \lambda F(d \alpha) d s \\
& \leq \lambda \int_{0}^{t} \int \sum_{k=2}^{2 m} C_{2 m}^{k} \mathrm{E}\left(\bar{\xi}_{s}^{2 m}\right)^{(2 m-k) /(2 m)} \alpha^{k}\left(\mathrm{E}\left[\xi_{s}^{0}\right]^{2 m}\right)^{k /(2 m)} F(d \alpha) d s
\end{aligned}
$$

Since

$$
\operatorname{Var} \eta_{t}=\frac{\sigma^{2}}{2 \gamma}\left[1-e^{-2 \gamma t}\right]
$$

\footnotetext{
${ }^{1}$ Editorial Note. In what follows, the authors use the $C$-notation for binomial coefficients; e.g., $C_{2 m}^{k}=\left(\begin{array}{c}2 m \\ k\end{array}\right)$.
} 
and the random variable

$$
\eta=\frac{\eta_{t}}{\sqrt{\frac{\sigma^{2}}{2 \gamma}\left[1-e^{-2 \gamma t}\right]}}
$$

has the standard Gaussian distribution, it follows that

$$
\begin{aligned}
\mathrm{E}\left[\xi_{t}^{0}\right]^{2 m} & =\mathrm{E} \exp \left\{-2 m[u \mu+(1-u) r] t+2 m \frac{u^{2} \sigma^{2}}{2} t-2 m u \eta_{t}\right\} \\
& =\exp \left\{-2 m[u \mu+(1-u) r] t+2 m \frac{u^{2} \sigma^{2}}{2} t\right\} \mathrm{E} \exp \left\{-2 m u \eta_{t}\right\} \\
& =\exp \left\{-2 m[u \mu+(1-u) r] t+2 m \frac{u^{2} \sigma^{2}}{2} t+2 m^{2} u^{2} \frac{\sigma^{2}}{2 \gamma}\left[1-e^{-2^{\gamma t}}\right]\right\} \\
& \leq \exp \left\{-2 m[u \mu+(1-u) r] t+m u^{2} \sigma^{2} t(1+2 m)\right\} .
\end{aligned}
$$

Relation (26) implies that

$$
\mathrm{E}\left|\bar{\xi}_{t}\right|^{2 m} \leq \lambda \int_{0}^{t} \sum_{k=2}^{2 m} C_{2 m}^{k} \mathrm{E}\left(\xi_{s}^{2 m}\right)^{(2 m-k) /(2 m)}\left[\mathrm{E} \varsigma_{i}^{2 m}\right]^{k /(2 m)}\left(\mathrm{E}\left[\xi_{s}^{0}\right]^{2 m}\right)^{k /(2 m)} d s
$$

in view of

$$
\int \alpha^{k} F(d \alpha) \leq\left[\int \alpha^{2 m} F(d \alpha)\right]^{k /(2 m)}=\left[\mathrm{E} \varsigma_{i}^{2 m}\right]^{k /(2 m)} .
$$

Now we find $0<\bar{u} \leq 1$ minimizing the right hand side of (27). It is easy to see that

$$
-2[\mu-r]+2 u^{*} \sigma^{2}(1+2 m)=0 .
$$

Thus the right hand side of (27) attains its minimum at the point

$$
u^{*}=\frac{[\mu-r]}{\sigma^{2}(1+2 m)} .
$$

We take $\bar{u}=u^{*}$ if $u^{*}<1$. In this case, the minimum of the right hand side of (27) is

$$
\mathrm{E}\left[\xi_{t}^{0}\right]^{2 m} \leq \exp \left\{-2 m\left[r+\frac{(\mu-r)^{2}}{2 \sigma^{2}(1+2 m)}\right] t\right\} .
$$

If $u^{*} \geq 1$, then $\mu-r \geq \sigma^{2}(1+2 m)$ and we get

$$
\begin{gathered}
\mu-\sigma^{2}(1+2 m) \geq r>0, \\
\mu-\frac{\sigma^{2}(1+2 m)}{2} \geq r+\frac{\sigma^{2}(1+2 m)}{2} .
\end{gathered}
$$

Thus the right hand side of (27) attains the minimum at $\bar{u}=1$, which corresponds to the investment of the whole capital to risky assets. Note that the minimum does not exceed the bound

$$
\exp \left\{-2 m\left[r+\frac{\sigma^{2}(1+2 m)}{2}\right] t\right\} \text {. }
$$

Therefore

$$
\mathrm{E}\left[\xi_{t}^{0}\right]^{2 m} \leq \exp \left\{-2 m\left[r+\frac{\sigma^{2}(1+2 m)}{2}\right] t\right\} .
$$

The latter relation together with (29) implies the bound

$$
\mathrm{E}\left[\xi_{t}^{0}\right]^{2 m} \leq \exp \{-2 m d t\}
$$

where

$$
d= \begin{cases}r+\frac{(\mu-r)^{2}}{2 \sigma^{2}(1+2 m)}, & \frac{[\mu-r]}{\sigma^{2}(1+2 m)}<1 \\ r+\frac{\sigma^{2}(1+2 m)}{2}, & \frac{[\mu-r]}{\sigma^{2}(1+2 m)} \geq 1\end{cases}
$$


It follows from (26) that

$$
\begin{aligned}
\sup _{0 \leq \tau \leq t} \mathrm{E}\left|\bar{\xi}_{\tau}\right|^{2 m} & \leq \lambda \int_{0}^{t} \sum_{k=2}^{2 m} C_{2 m}^{k} \sup _{0 \leq \tau \leq s} \mathrm{E}\left(\bar{\xi}_{\tau}^{2 m}\right)^{(2 m-k) /(2 m)}\left[\mathrm{E} \varsigma_{i}^{2 m}\right]^{k /(2 m)} \exp \{-k d s\} d s \\
& \leq \sum_{k=2}^{2 m} \lambda C_{2 m}^{k} \sup _{0 \leq \tau \leq t} \mathrm{E}\left(\bar{\xi}_{\tau}^{2 m}\right)^{(2 m-k) /(2 m)}\left[\mathrm{E} \varsigma_{i}^{2 m}\right]^{k /(2 m)} \frac{1-\exp \{-d k t\}}{d k} \\
& \leq \sum_{k=2}^{2 m} \lambda C_{2 m}^{k} \sup _{0 \leq \tau \leq t} \mathrm{E}\left(\bar{\xi}_{\tau}^{2 m}\right)^{(2 m-k) /(2 m)}\left[\mathrm{E} \varsigma_{i}^{2 m}\right]^{k /(2 m)} \frac{1}{d k} .
\end{aligned}
$$

Let

$$
x=\left(\sup _{0 \leq \tau \leq t} \mathrm{E}\left|\bar{\xi}_{\tau}\right|^{2 m}\right)^{1 /(2 m)} .
$$

Then relation (30) implies

$$
x^{2 m} \leq \sum_{k=2}^{2 m} \lambda C_{2 m}^{k} x^{2 m-k} b^{k} \frac{1}{d k} \leq b^{2} \lambda C_{2 m}^{2} \frac{1}{2 d} \sum_{k=2}^{2 m} C_{2 m-2}^{k-2} x^{2 m-2-(k-2)} b^{k-2} \frac{C_{2 m}^{k}}{C_{2 m}^{2} C_{2 m-2}^{k-2}} .
$$

Since

$$
\frac{C_{2 m}^{k}}{C_{2 m}^{2} C_{2 m-2}^{k-2}}=\frac{2 m ! 2 !(2 m-2) !(k-2) !(2 m-k) !}{k !(2 m-k) ! 2 m !(2 m-2) !}=\frac{2 !}{(k-1)} \leq 1, \quad k \geq 2,
$$

we derive from (31) that

$$
\begin{aligned}
x^{2 m} & \leq b^{2} \lambda C_{2 m}^{2} \frac{1}{2 d} \sum_{k=2}^{2 m} C_{2 m-2}^{k-2} x^{2 m-2-(k-2)} b^{k-2} \\
& =b^{2} \lambda C_{2 m}^{2} \frac{1}{2 d} \sum_{i=0}^{2 m-2} C_{2 m-2}^{i} x^{2 m-2-i} b^{i}=b^{2} \lambda C_{2 m}^{2} \frac{1}{2 d}(b+x)^{2 m-2} \\
& =b^{2} \lambda C_{2 m}^{2} \frac{1}{2 d} x^{2 m-2}\left(1+\frac{b}{x}\right)^{2 m-2} .
\end{aligned}
$$

If $x>0$, we deduce from (32) that

$$
x^{2} \leq b^{2} \lambda C_{2 m}^{2} \frac{1}{2 d}\left(1+\frac{b}{x}\right)^{2 m-2},
$$

whence

$$
x \leq b \sqrt{\frac{\lambda C_{2 m}^{2}}{2 d}}\left(1+\frac{b}{x}\right)^{m-1} .
$$

Consider the graphs of the functions $y=x$ and $y=b \sqrt{\frac{\lambda C_{2 m}^{2}}{2 d}}\left(1+\frac{b}{x}\right)^{m-1}$ (see Figure 1).

Let $R>0$ be a solution of the equation

$$
x=b \sqrt{\frac{\lambda C_{2 m}^{2}}{2 d}}\left(1+\frac{b}{x}\right)^{m-1} ;
$$

that is,

$$
R=b \sqrt{\frac{\lambda C_{2 m}^{2}}{2 d}}\left(1+\frac{b}{R}\right)^{m-1}
$$




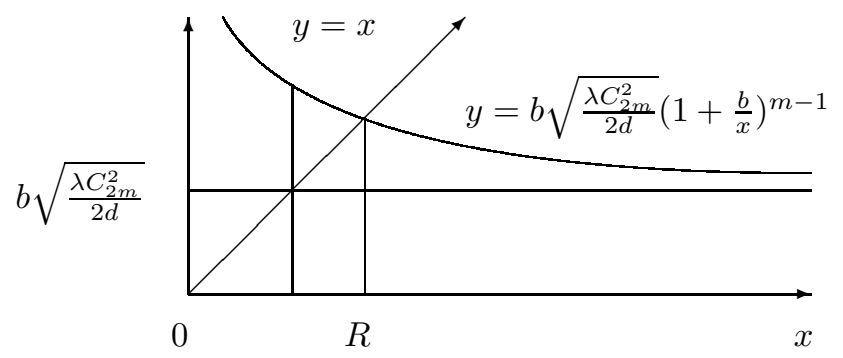

FIGURE 1.

Then $0 \leq x \leq R$ is a solution of inequality (33). It is easy to see that

$$
R>b \sqrt{\frac{\lambda C_{2 m}^{2}}{2 d}} .
$$

The function

$$
y=b \sqrt{\frac{\lambda C_{2 m}^{2}}{2 d}}\left(1+\frac{b}{x}\right)^{m-1}
$$

is increasing; thus

$$
R<b \sqrt{\frac{\lambda C_{2 m}^{2}}{2 d}}\left(1+\sqrt{\frac{2 d}{\lambda C_{2 m}^{2}}}\right)^{m-1}
$$

as $x \rightarrow 0$. This implies the following bound:

$$
\sup _{0 \leq t<+\infty} \mathrm{E}\left|\bar{\xi}_{\tau}\right|^{2 m} \leq b^{2 m}\left(\frac{\lambda C_{2 m}^{2}}{2 d}\right)^{m}\left(\left[1+\sqrt{\frac{2 d}{\lambda C_{2 m}^{2}}}\right]^{2}\right)^{m(m-1)} .
$$

Theorem 1 is proved.

Remark. It is easy to see that

$$
\begin{aligned}
\left(\left[1+\sqrt{\frac{2 d}{\lambda C_{2 m}^{2}}}\right]^{2}\right)^{(m-1)} & =\left[1+\frac{2 \sqrt{d}}{\sqrt{\lambda m(2 m-1)}}\right]^{2(m-1)} \\
& =\left[1+\frac{2 \sqrt{d}}{\sqrt{\lambda m(2 m-1)}}\right]^{\frac{\sqrt{\lambda m(2 m-1)}}{2 \sqrt{d}} \frac{4(m-1) \sqrt{d}}{\sqrt{\lambda m(2 m-1)}} \underset{m \rightarrow+\infty}{\longrightarrow} e^{2 \sqrt{2 d \lambda^{-1}}}}
\end{aligned}
$$

that is,

$$
b^{2 m}\left(\frac{\lambda C_{2 m}^{2}}{2 d}\right)^{m}\left(\left[1+\sqrt{\frac{2 d}{\lambda C_{2 m}^{2}}}\right]^{2}\right)^{m(m-1)} \approx\left(b^{2} \frac{\lambda m(2 m-1)}{2 d} e^{2 \sqrt{2 d \lambda^{-1}}}\right)^{m}
$$

as $m \rightarrow+\infty$.

\section{INEQUALITIES FOR THE RUIN PROBABILITY OF AN INSURANCE COMPANY} OVER THE TIME INTERVAL $[0,+\infty)$

We distinguish between the three cases: $\bar{u}=0, \bar{u}=u^{*}$, and $\bar{u}=1$ that correspond to different investment strategies. 
We follow the idea of 9 . Let $\bar{u}=0$. This means that the entire capital is invested in bonds. Assume that $c-a \lambda>0$. We have

$$
\xi_{t}^{0}=\exp \{-r t\} \leq 1 .
$$

Let

$$
\psi(t, z)=\lambda \int_{0}^{t} \int\left[e^{z \alpha \xi_{s}^{0}}-1-z \alpha \xi_{s}^{0}\right] F(d \alpha) d s-\int_{0}^{t} z \xi_{s}^{0}(c-a \lambda) d s
$$

where $z>0$ and $\xi_{s}^{0}>0$. We also consider the function

$$
\varphi(\beta)=\lambda \int\left[e^{\beta \alpha}-1-\beta \alpha\right] F(d \alpha)-\beta(c-a \lambda)
$$

for $\beta \geq 0$. It is easy to see that $\varphi(0)=0$. Since

$$
\varphi^{\prime}(\beta)=\lambda \int \alpha\left[e^{\beta \alpha}-1\right] F(d \alpha)-(c-a \lambda),
$$

we have $\varphi^{\prime}(0)=-(c-a \lambda)<0$. This means that the function $\varphi(\beta)$ starts from zero and decreases (in particular, $\varphi$ is negative for some period of time). It is also obvious that the derivative

$$
\varphi^{\prime}(\beta)=\lambda \int \alpha\left[e^{\beta \alpha}-1\right] F(d \alpha)-(c-a \lambda)
$$

is positive after some $\beta>0$. Thus the function $\varphi(\beta)$ has a root $R$ different of 0 ; that is,

$$
\lambda \int\left[e^{R \alpha}-1-R \alpha\right] F(d \alpha)-R(c-a \lambda)=0 .
$$

Then

$$
\varphi(\beta)=\lambda \int\left[e^{\beta \alpha}-1-\beta \alpha\right] F(d \alpha)-\beta(c-a \lambda) \leq 0
$$

for $0<\beta \leq R$. Inequalities (37) and (40) imply that $\psi(t, z) \leq 0$ for $z \leq R$. Let $z=R$. Considering (21) we get

$$
\begin{aligned}
& \mathrm{P}\left\{\inf _{0 \leq t \leq T} \xi_{t} \leq 0\right\} \\
& \quad=\mathrm{P}\left\{\inf _{0 \leq t \leq T}\left[\xi_{t}^{0}\right]^{-1}\left[\xi_{0}+\int_{0}^{t} \xi_{s}^{0}\left((c-a \lambda) d s-\int y \tilde{\nu}(d y, d s)\right)\right] \leq 0\right\} \\
&=\mathrm{P}\left\{\inf _{0 \leq t \leq T}\left[\xi_{0}+\int_{0}^{t} \xi_{s}^{0}\left((c-a \lambda) d s-\int y \tilde{\nu}(d y, d s)\right)\right] \leq 0\right\} \\
&=\mathrm{P}\left\{\operatorname { s u p } _ { 0 \leq t \leq T } \left[R \int_{0}^{t} \int \xi_{s}^{0} y \tilde{\nu}(d y, d s)\right.\right. \\
& \leq \mathrm{P}\left\{\operatorname { s u p } _ { 0 \leq t \leq T } \operatorname { e x p } \left[R \int_{0}^{t} \int \xi_{s}^{0} y \tilde{\nu}(d y, d s)\right.\right. \\
& \leq e^{-R \xi_{0}} .
\end{aligned}
$$


The latter inequality follows from the Kolmogorov inequality, since the stochastic process

$$
\rho(t)=\exp \left[R \int_{0}^{t} \int \xi_{s}^{0} y \tilde{\nu}(d y, d s)-\lambda \int_{0}^{t} \int\left[e^{R y \xi_{s}^{0}}-1-R y \xi_{s}^{0}\right] F(d y) d s\right]
$$

is a martingale. If $\bar{u}=u^{*}$ or $\bar{u}=1$ in the case of $c \geq a \lambda$, then

$$
\begin{aligned}
\mathrm{P}\left\{\inf _{0 \leq t \leq T} \xi_{t} \leq 0\right\} & =\mathrm{P}\left\{\inf _{0 \leq t \leq T}\left[\xi_{t}^{0}\right]^{-1}\left[\xi_{0}+\int_{0}^{t} \xi_{s}^{0}\left((c-a) d s-\int y \tilde{\nu}(d y, d s)\right)\right] \leq 0\right\} \\
& =\mathrm{P}\left\{\inf _{0 \leq t \leq T}\left[\xi_{0}+\int_{0}^{t} \xi_{s}^{0}\left((c-a) d s-\int y \tilde{\nu}(d y, d s)\right)\right] \leq 0\right\} \\
& =\mathrm{P}\left\{\sup _{0 \leq t \leq T}\left[\int_{0}^{t} \int \xi_{s}^{0} y \tilde{\nu}(d y, d s)\right] \geq \xi_{0}+\int_{0}^{T} \xi_{s}^{0}(c-a \lambda) d s\right\} \\
& \leq \mathrm{E}_{\xi^{0}} \frac{\mathrm{E}_{\nu}\left|\int_{0}^{T} \int \xi_{s}^{0} y \tilde{\nu}(d y, d s)\right|^{2 m}}{\left[\xi_{0}+\int_{0}^{T} \xi_{s}^{0}(c-a \lambda) d s\right]^{2 m}} \leq \frac{\mathrm{E}_{\xi^{0}} \mathrm{E}_{\nu}\left|\int_{0}^{T} \int \xi_{s}^{0} y \tilde{\nu}(d y, d s)\right|^{2 m}}{\left[\xi_{0}\right]^{2 m}}
\end{aligned}
$$

since the Wiener process $W_{t}$ and Poisson measure $\nu(A, t)$ are independent. Taking (25) into account we obtain

$$
\begin{aligned}
\mathrm{P}\left\{\inf _{0 \leq t \leq T} \xi_{t} \leq 0\right\} & \leq \frac{\mathrm{E}_{\xi 0} \mathrm{E}_{\nu}\left|\int_{0}^{T} \int \xi_{s}^{0} y \tilde{\nu}(d y, d s)\right|^{2 m}}{\left[\xi_{0}\right]^{2 m}} \\
& \leq \frac{b^{2 m}\left(\frac{\lambda C_{2 m}^{2}}{2 d}\right)^{m}\left(\left[1+\sqrt{\frac{2 d}{\lambda C_{2 m}^{2}}}\right]^{2}\right)^{m(m-1)}}{\left[\xi_{0}\right]^{2 m}}
\end{aligned}
$$

where $d= \begin{cases}r+\frac{(\mu-r)^{2}}{2 \sigma^{2}(1+2 m)}, & \frac{[\mu-r]}{\sigma^{2}(1+2 m)}<1 \\ r+\frac{\sigma^{2}(1+2 m)}{2}, & \frac{[\mu-r]}{\sigma^{2}(1+2 m)} \geq 1\end{cases}$

Passing to the limit as $T \rightarrow+\infty$ we derive from (41) that

$$
\mathrm{P}\left\{\inf _{0 \leq t<+\infty} \xi_{t} \leq 0\right\}=\lim _{T \rightarrow+\infty} \mathrm{P}\left\{\inf _{0 \leq t \leq T} \xi_{t} \leq 0\right\} \leq e^{-R \xi_{0}}
$$

(see [8]), while (42) implies that

$$
\begin{aligned}
\mathrm{P}\left\{\inf _{0 \leq t<+\infty} \xi_{t} \leq 0\right\} & =\lim _{T \rightarrow+\infty} \mathrm{P}\left\{\inf _{0 \leq t \leq T} \xi_{t} \leq 0\right\} \\
& \leq \frac{b^{2 m}\left(\frac{\lambda C_{2 m}^{2}}{2 d}\right)^{m}\left(\left[1+\sqrt{\frac{2 d}{\lambda C_{2 m}^{2}}}\right]^{2}\right)^{m(m-1)}}{\left[\xi_{0}\right]^{2 m}} .
\end{aligned}
$$

Thus we have proved the following result.

Theorem 2. Let $\xi_{0}$ be the initial capital of an insurance company dealing in a BSmarket. Let the evolution of a risky asset be described by relation (2). Let $0<r<\mu$ where $r$ is the bank interest. Let the accumulated claim process be described by the compound Poisson process (14) where $\lambda>0$ is the intensity of a standard Poisson process $Z_{t}$ modelling the number of claims over the interval $[0, t]$. Put $F(x)=\mathrm{P}\left\{\varsigma_{k}<x\right\}$ where $0<\varsigma_{k}$ are independent identically distributed random variables that do not depend on the Poisson process $Z_{t}$ and have finite exponential moments. Put $\mathrm{E}_{\varsigma_{k}}=a$. Then 
1) if $c>a \lambda$ and the entire capital is invested in bonds, that is $\bar{u}=0$, then the ruin probability admits the following estimate:

$$
\mathrm{P}\left\{\inf _{0 \leq t<+\infty} \xi_{t} \leq 0\right\} \leq e^{-R \xi_{0}}
$$

where $R>0$ satisfies the equation

$$
\lambda \int\left[e^{R \alpha}-1-R \alpha\right] F(d \alpha)-R(c-a \lambda)=0 ;
$$

2) if $\mathrm{E} \varsigma_{i}^{2 m}<+\infty, c \geq a \lambda$, and $[\mu-r] /\left(\sigma^{2}(1+2 m)\right) \geq 1$, that is, the entire capital is invested in stocks and $\bar{u}=1$, then the ruin probability admits the following estimate:

$$
\mathrm{P}\left\{\inf _{0 \leq t<+\infty} \xi_{t} \leq 0\right\} \leq \frac{b^{2 m}\left(\frac{\lambda C_{2 m}^{2}}{2 d}\right)^{m}\left(\left[1+\sqrt{\frac{2 d}{\lambda C_{2 m}^{2}}}\right]^{2}\right)^{m(m-1)}}{\left[\xi_{0}\right]^{2 m}}
$$

where

$$
d=r+\frac{\sigma^{2}(1+2 m)}{2}, \quad b=\left[\mathrm{E} \varsigma_{i}^{2 m}\right]^{1 /(2 m)} ;
$$

3) if $\mathrm{E} \varsigma_{i}^{2 m}<+\infty, c \geq a \lambda$, and $[\mu-r] /\left(\sigma^{2}(1+2 m)\right)=u^{*}<1$, that is, $\bar{u}=u^{*}$ and the fraction $\bar{u} \cdot 100 \%$ of the initial capital is invested in the risky asset and the rest of the capital is used to purchase bonds, then the ruin probability can be estimated as follows:

$$
\mathrm{P}\left\{\inf _{0 \leq t<+\infty} \xi_{t} \leq 0\right\} \leq \frac{b^{2 m}\left(\frac{\lambda C_{2 m}^{2}}{2 d}\right)^{m}\left(\left[1+\sqrt{\frac{2 d}{\lambda C_{2 m}^{2}}}\right]^{2}\right)^{m(m-1)}}{\left[\xi_{0}\right]^{2 m}}
$$

where

$$
d=r+\frac{(\mu-r)^{2}}{2 \sigma^{2}(1+2 m)}, \quad b=\left[\mathrm{E} \varsigma_{i}^{2 m}\right]^{1 /(2 m)} .
$$

\section{Concluding Remarks}

It is well known that if an insurance company invests all the capital in bonds, then the Lundberg bound holds for the ruin probability over an infinite interval:

$$
\mathrm{P}\left\{\inf _{0 \leq t<+\infty} \xi_{t} \leq 0\right\} \leq e^{-R \xi_{0}}
$$

where $R>0$ is a nonzero solution of the equation $\lambda \int e^{x y} d F(y)=c x+\lambda$. Some similar problems are considered in 10 for an insurance company dealing in a $B S$-market for the case where the evolution of the price of a risky asset is described by the classical models. The Wiener process is the basic tool used in [10 to describe the dynamics of the price of a risky asset; that is, the Samuelson model is studied in 10. The probability of ruin of an insurance company is considered in the paper [10] for the case where either the entire capital is invested in bonds or the capital is divided for mixing investments. It is shown in [9] that if the capital is invested in bonds, then the Lundberg bound (49) holds. In the general case as well as in the case of the Samuelson model, inequality (49) is not yet proved.

The case considered in this paper is of a more complicated nature, since the underlying stochastic process describing the evolution of the capital of a company is more complicated. 
One of the important problems for the above model is the estimation of the probability of ruin of an insurance company for which the constant $c>0$ is equal to

$$
a \lambda=\lambda \mathrm{E}_{k},
$$

which corresponds to the zero load. If

$$
\bar{u}=\frac{\mu-r}{\sigma^{2}(1+2 m)}<1, \quad m=0,1, \ldots,
$$

that is, the fraction $\frac{\mu-r}{\sigma^{2}(1+2 m)} \cdot 100 \%$ of the capital is invested in the risky asset and the rest is invested in bonds, then

$$
\begin{aligned}
\mathrm{E}\left[\xi_{t}^{0}\right]^{-1} & =\mathrm{E} \exp \left\{[\bar{u} \mu+(1-\bar{u}) r] t-\frac{\bar{u}^{2} \sigma^{2}}{2} t+\bar{u} \eta_{t}\right\} \\
& =\exp \left\{[\bar{u} \mu+(1-\bar{u}) r] t-\frac{\bar{u}^{2} \sigma^{2}}{2} t+\bar{u}^{2} \frac{\sigma^{2}}{\gamma}\left[1-e^{-2^{\gamma t}}\right]\right\} \\
& \geq \exp \left\{r t+\frac{(\mu-r)^{2}}{\sigma^{2}} \frac{m}{(1+2 m)^{2}} t\right\}>1 .
\end{aligned}
$$

If $t>s$, then

$$
\begin{aligned}
\mathrm{E}\left[\xi_{t}^{0}\right]^{-1} \xi_{s}^{0} & =\mathrm{E} \exp \left\{[\bar{u} \mu+(1-\bar{u}) r](t-s)-\frac{\bar{u}^{2} \sigma^{2}}{2}(t-s)+\bar{u}\left(\eta_{t}-\eta_{s}\right)\right\} \\
& =\exp \left\{[\bar{u} \mu+(1-\bar{u}) r](t-s)-\frac{\bar{u}^{2} \sigma^{2}}{2}(t-s)+\frac{\bar{u}^{2} \operatorname{Var}\left(\eta_{t}-\eta_{s}\right)}{2}\right\} \\
& \geq \exp \left\{\left(r+\frac{(\mu-r)^{2}}{\sigma^{2}} \frac{m}{(1+2 m)^{2}}\right)(t-s)\right\}>1
\end{aligned}
$$

whence

for $t>0$. If

$$
\mathrm{E} \xi_{t}=\int_{0}^{t} \mathrm{E}\left[\xi_{t}^{0}\right]^{-1} \xi_{s}^{0}(c-a \lambda) d s+\xi_{0}>(c-a \lambda) t+\xi_{0}
$$

$$
\frac{\mu-r}{\sigma^{2}(1+2 m)} \geq 1, \quad m=0,1, \ldots,
$$

that is, if the entire capital is invested in the risky asset (obviously, $\mu \geq \sigma^{2}(1+2 m)$ in this case), then

$$
\mathrm{E}\left[\xi_{t}^{0}\right]^{-1}=\mathrm{E} \exp \left\{\mu t-\frac{\sigma^{2}}{2} t+\eta_{t}\right\}=\exp \left\{\mu t-\frac{\sigma^{2}}{2} t+\frac{\sigma^{2}}{\gamma}\left[1-e^{-{ }^{2 \gamma t}}\right]\right\}>1
$$

for $t>0$. If $t>s$, then

$$
\begin{aligned}
\mathrm{E}\left[\xi_{t}^{0}\right]^{-1} \xi_{s}^{0} & =\mathrm{E} \exp \left\{\mu(t-s)-\frac{\sigma^{2}}{2}(t-s)+\eta_{t}-\eta_{s}\right\} \\
& \geq \exp \left\{\mu(t-s)-\frac{\sigma^{2}}{2}(t-s)\right\}>1
\end{aligned}
$$

and

$$
\mathrm{E} \xi_{t}=\mathrm{E}\left[\xi_{t}^{0}\right]^{-1} \int_{0}^{t}(c-a \lambda) d s+\xi_{0}>(c-a \lambda) t+\xi_{0}
$$

Relations (50) and (51) imply that the mean capital of an insurance company dealing in a $B S$-market is greater than that of a company investing the capital only in bonds. This result does not hold with probability 1 (in which case we would obtain bound (49)). Inequalities (45) and (47) of Theorem 2 provide bounds for the ruin probability of an 
insurance company dealing in a $B S$-market. The case of a zero load is also possible; that is, $c>0$ may equal $a \lambda=\lambda \mathrm{E} \varsigma_{k}$.

\section{BIBLIOGRAPHY}

1. A. V. Baev and B. V. Bondarev, Ornstein-Uhlenbeck process and its applications in problems of actuarial mathematics, Applied statistics. Actuarial and Financial Mathematics (2002), no. 1, $3-28$.

2. P. A. Samuelson, Proof that properly anticipated prices fluctuate randomly, Industrial Management Review 6 (1965), 41-49.

3. A. N. Shiryaev, Essentials of Stochastic Finance. Facts, Models, Theory, Fazis, Moscow, 1998; English transl., World Scientific Publishing Co., Inc., River Edge, NJ, 1999. MR1695318 (2000e:91085)

4. A. V. Skorokhod, Lectures on the Theory of Stochastic Processes, Lybid', Kyiv, 1990; English transl., VSP/TViMS, Utrecht, Netherlands/Kiev, Ukraine, 1996. MR.1452108(99d:60001)

5. B. V. Bondarev, Mathematical Models in Insurance, Apeks, Donetsk, 2002. (Russian)

6. I. I. Gikhman and A. V. Skorokhod, Stochastic Differential Equations and their Applications, Naukova dumka, Kiev, 1982. (Russian) MR678374 (84j:60003)

7. I. I. Gikhman and A. V. Skorokhod, Stochastic Differential Equations, Naukova Dumka, Kiev, 1968; English transl., Springer-Verlag, New York-Heidelberg, 1972. MR0263172 (41:7777) MR 0346904 (49:11625)

8. I. I. Gikhman, A. V. Skorokhod, and M. I. Yadrenko, Probability Theory and Mathematical Statistics, Vyshcha Shkola, Kiev, 1988. (Russian)

9. A. V. Baev and B. V. Bondarev, An insurance company dealing in a $(B, S)$ market, Applied statistics. Actuarial and Financial Mathematics (2003), no. 1-2, 11-26.

10. A. V. Melnikov, Risk Analysis in Finance and Insurance, Ankil, Moscow, 2001; English transl., Chapman \& Hall/CRC, Boca Raton, FL, 2004. MR2013235 (2004i:91004)

Department of Probability Theory and Mathematical Statistics, Faculty for Mathematics, Donetsk State University, Universitets'ka Street 24, 83055 Donetsk, Ukraine

E-mail address: tv@matfak.dongu.donetsk.ua

Department of Probability Theory and Mathematical Statistics, Faculty for Mathematics, Donetsk State University, Universitets'ka Street 24, 83055 Donetsk, Ukraine

E-mail address: bvbondarev@cable.netlux.org

Received 11/JAN/2005

Translated by V. V. SEMENOV 